\title{
ARTICLE Discovery and characterization of natural products as novel indoleamine 2,3-dioxygenase 1 inhibitors through high-throughput screening
}

Wei Guo ${ }^{1,2}$, Sheng Yao ${ }^{3,4}$, Pu Sun ${ }^{1,2}$, Tian-biao Yang ${ }^{4}$, Chun-ping Tang ${ }^{3,4}$, Ming-yue Zheng ${ }^{4}$, Yang Ye ${ }^{3,4,5}$ and Ling-hua Meng ${ }^{1,2}$

Indoleamine 2,3-dioxygenase 1 (IDO1) is emerging as a promising therapeutic target for the treatment of malignant tumors characterized by dysregulated tryptophan metabolism. However, the antitumor efficacy of existing small-molecule IDO1 inhibitors is still unsatisfactory, and the underlying mechanism remains largely undefined. To identify novel IDO1 inhibitors, an in-house natural product library of 2000 natural products was screened for inhibitory activity against recombinant human IDO1. Highthroughput fluorescence-based screening identified 79 compounds with inhibitory activity $>30 \%$ at $20 \mu \mathrm{M}$. Nine natural products were further confirmed to inhibit IDO1 activity by $>30 \%$ using Ehrlich's reagent reaction. Compounds 2, 7, and $\mathbf{8}$ were demonstrated to inhibit IDO1 activity in a cellular context. Compounds $\mathbf{2}$ and $\mathbf{7}$ were more potent against IDO1 than TDO2 in the enzymatic assay. The kinetic studies showed that compound $\mathbf{2}$ exhibited noncompetitive inhibition, whereas compounds $\mathbf{7}$ and $\mathbf{8}$ were graphically well matched with uncompetitive inhibition. Compounds $\mathbf{7}$ and $\mathbf{8}$ were found to bind to the ferric-IDO1 enzyme. Docking stimulations showed that the naphthalene ring of compound $\mathbf{8}$ formed "T-shaped" $\pi-\pi$ interactions with Phe-163 and that the 6-methyl-naphthalene group formed additional hydrophobic interactions with IDO1. Compound $\mathbf{8}$ was identified as a derivative of tanshinone, and preliminary SAR analysis indicated that tanshinone derivatives may be promising hits for the development of IDO1 inhibitors. This study provides new clues for the discovery of IDO1/TDO2 inhibitors with novel scaffolds.

Keywords: indoleamine 2,3-dioxygenase 1 inhibitor; natural product library; high-throughput fluorescence-based screening

Acta Pharmacologica Sinica (2020) 41:423-431; https://doi.org/10.1038/s41401-019-0246-4

\section{INTRODUCTION}

Indoleamine 2,3-dioxygenase (IDO) and tryptophan 2,3-dioxygenase 2 (TDO2) are intracellular heme-containing metalloproteins that initiate the first and the rate-limiting steps of tryptophan breakdown along the kynurenine pathway [1]. IDO is ubiquitously expressed in many types of cells and tissues, with the highest expression occurring in antigen-presenting cells, such as macrophages and dendritic cells. Two homologs, IDO1 and IDO2, have been found in the IDO family, and IDO1 is by far the better characterized of the two, and it has a central role in mediating immune privilege and preventing T-cell-driven rejection of allogeneic fetuses during pregnancy [2]. Although the sequence similarity between human IDO1 and TDO2 is poor (16\%), the high similarity of their catalytic domains has been revealed by protein crystallography studies. Although TDO2 is specific for metabolizing tryptophan to kynurenine, IDO1 recognizes a broad range of indole-containing substrates, including the neurotransmitter melatonin. IDO1 is ubiquitously expressed in many tissues and cells, including endothelial cells and cells that in part constitute the tumor microenvironment, such as fibroblasts, macrophages, myeloid-derived suppressor cells, and dendritic cells. In contrast, TDO2 is predominantly expressed in the liver, where it has a key role in maintaining the systemic homeostasis of the tryptophan levels [3]. The expression of IDO1 is greatly elevated in multiple types of human cancer and is correlated with a poor prognosis [4-6]. Recently, IDO1 has been shown to play an important role in the process of immune evasion by tumors [7]. The IDO-mediated depletion of local tryptophan levels and the production of toxic metabolites results in the suppression of T-cell responses and the enhancement of immunosuppression mediated by regulatory $T$ cells $[8,9]$.

As such, IDO1 has emerged as a promising therapeutic target, prompting searches for highly active inhibitors. The landmark competitive inhibitor 1-methyl-L-tryptophan (1-L-MT) was identified in the early $1990 \mathrm{~s}$ and has a reported $K \mathrm{i}$ of $34 \mu \mathrm{M}$. However, $1-L-M T$ is a rather poor IDO1 inhibitor when tested in IDO1expressing human tumor cells, as 1-L-MT concentrations $>200 \mu \mathrm{M}$ are required to block IDO1-mediated tryptophan degradation by $50 \%$ [10]. The X-ray crystal structure of human IDO1 in a complex with an inhibitor (4-phenylimidazole or cyanide) was revealed in

\footnotetext{
${ }^{1}$ Division of Anti-tumor Pharmacology, Shanghai Institute of Materia Medica, Chinese Academy of Sciences, Shanghai 201203, China; ${ }^{2}$ University of Chinese Academy of Sciences, Beijing 100049, China; ${ }^{3}$ Department of Natural Products Chemistry, Shanghai Institute of Materia Medica, Chinese Academy of Sciences, Shanghai 201203 , China; ${ }^{4}$ State Key

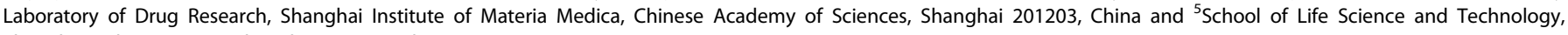
ShanghaiTech University, Shanghai 201203, China
}

Correspondence: Yang Ye (yye@mail.shcnc.ac.cn) or Ling-hua Meng (Ihmeng@simm.ac.cn)

These authors contributed equally: Wei Guo, Sheng Yao

Received: 25 January 2019 Accepted: 5 May 2019

Published online: 13 June 2019 
2006 and facilitated the discovery of new IDO1 inhibitors [11]. Recently, several potent IDO1 inhibitors have been identified by unbiased screening [12, 13], structure-based modification [14, 15], and in silico drug design $[15,16]$. However, only six compounds, 1-methyl-D-tryptophan (1-D-MT), hydroxylamidine INCB024360, NLG802, BMS-986205, SHR9146, LY3381916, and PF-06840003, are currently in clinical trials. Therefore, the number and structures of IDO1 inhibitors in clinical trials are very limited, and new inhibitors with diverse structures and improved activities are of great interest.

Herein, we screened an in-house natural product library for novel inhibitors of IDO1. The screening led to the discovery that compounds 2, 7, and $\mathbf{8}$ showed inhibitory potencies in the micromolar range. Detailed kinetic studies were performed and revealed that compound $\mathbf{2}$ exhibited noncompetitive inhibition, whereas compounds $\mathbf{7}$ and $\mathbf{8}$ showed uncompetitive inhibition. Compounds $\mathbf{7}$ and $\mathbf{8}$ were shown to interact with the ferric form of IDO1. Preliminary SARs were drawn from the analogs of compound $\mathbf{8}$ and corroborated the putative binding orientation suggested by molecular docking.

\section{MATERIALS AND METHODS}

Materials

Methylene Blue hydrate (\#28514), L-tryptophan (\#T0254), DMSO (\#D2650), and p-dimethylaminobenzaldehyde (\#D2004) were purchased from Sigma (St. Louis, MO, USA). Catalase, bovine hemin, and ascorbic acid were purchased from Sangon (Shanghai, China). Piperidine and TCA were obtained from SCRC (Shanghai, China). INCB024360 and Selleck Customized Library-Z100688 were purchased from Selleck Chemicals (Houston, TX, USA). The natural product library was constructed by the Department of Natural Products Chemistry, Shanghai Institute of Materia Medica, Chinese Academy of Sciences. Cell Counting Kit-8(\#CK04) was purchased from Dojindo (Kumamoto, Japan). Recombinant IDO1 and TDO2 were expressed and purified in our laboratory as described previously [17]. The plasmids pET28a-hIDO1, pET28a-hTDO2, pcDNA3.1-hIDO1, and pcDNA3.1-TDO2 were synthesized by Synbio Technologies (Suzhou, China).

Cell culture

HEK 293 cell line was obtained from ATCC (Manassas, VA, USA). Cells were grown in Dulbecco's modified Eagle's medium (Corning, New York, USA) supplemented with $10 \%$ fetal bovine serum (Gibco, New York, USA) in $5 \% \mathrm{CO}_{2}$ at $37^{\circ} \mathrm{C}$.

\section{Expression and purification of rhIDO1 and TDO2}

Recombinant human IDO1 (rhIDO1) was expressed and purified from Escherichia coli transformed with pET28a-hIDO1 and pET28ahTDO2 plasmids as described previously [17]. The elution buffer for the gel filtration was $25 \mathrm{mM}$ Tris $(\mathrm{pH} 7.4)$ with $5 \%$ glycerol. The Soret's ratio $\left(A_{404} / A_{280}\right)$ of purified protein was 1.9-2.2.

Enzymatic assays of IDO1 and TDO2

The effects of the test compounds on the enzymatic activity of IDO1 were determined as previously described with minor modifications [18]. In brief, the reaction mixture $(30 \mu \mathrm{L})$ containing potassium phosphate $(100 \mathrm{mM}, \mathrm{pH} 6.5)$, ascorbic acid neutralized with $\mathrm{NaOH}(40 \mathrm{mM})$, catalase $(200 \mu \mathrm{g} / \mathrm{mL})$, methylene blue $(20 \mathrm{mM})$, and rhIDO1 or rhTDO2 $(0.05 \mu \mathrm{M})$ was added to a solution $(60 \mu \mathrm{L})$ containing the substrate ( $L$-tryptophan, $250 \mu \mathrm{M})$ and test compounds at the desired concentration. The reaction was carried out at $37^{\circ} \mathrm{C}$ for $30 \mathrm{~min}$ and stopped by adding $45 \mu \mathrm{L}$ of $30 \%(\mathrm{w} / \mathrm{v})$ trichloroacetic acid. After being incubated at $65^{\circ} \mathrm{C}$ for $15 \mathrm{~min}$, the reaction mixture was centrifuged at $12000 \mathrm{r} / \mathrm{min}$ for $10 \mathrm{~min}$. The supernatant $(100 \mu \mathrm{L})$ was transferred into a 96 -well microplate and mixed with $100 \mu \mathrm{L}$ of $2 \%(\mathrm{w} / \mathrm{v})$ p-dimethylaminobenzaldehyde (pDMAB) in acetic acid. The yellow pigment derived from kynurenine was measured at $492 \mathrm{~nm}$ using a SpectraMax Plus 384 microplate reader (Molecular Devices, Sunnyvale, CA). The data were analyzed using the enzyme kinetics module in SigmaPlot, version 10 .

The fluorescent enzymatic assay was carried out in 384-well plates as described previously [19] with minor modifications. The reaction was carried out in the presence of IDO1 holoenzyme, which consumed no more than $\sim 30 \%$ of the initial $L$-Trp over $30 \mathrm{~min}$ and was terminated by the addition of piperidine (200 mM, final concentration). The plate was incubated at $65^{\circ} \mathrm{C}$ for $15 \mathrm{~min}$ and left at room temperature for $60 \mathrm{~min}$ prior to measurement with a SYNERGY H1 microplate reader (Bioteck, Winooski, VT, USA). The inhibitory rate was calculated using the formula $\left(O D_{\text {control }}-O D_{\text {blank }}\right)-\left(O D_{\text {treatment }}-O D_{\text {blank }}\right) /\left(O D_{\text {control }}-\right.$ $\left.O D_{\text {blank }}\right) \times 100 \%$. The $I C_{50}$ values were determined by nonlinear regression analysis with Prism 4 software (GraphPad Software Inc., San Diego, CA, USA). The $Z^{\prime}$ factor of the assay was calculated using the formula $\left[\left(O D_{\text {mean }}\right.\right.$ control $\left.-3 S D_{\text {control }}\right)-\left(O D_{\text {mean }}\right.$ blank + $\left.\left.3 S D_{\text {blank }}\right)\right] /\left(O D_{\text {mean control }}-O D_{\text {mean blank }}\right)$.

\section{Cell-based assay of IDO1 and TDO2 activities}

HEK 293 cells were seeded in a six-well plate at a density of $5 \times 10^{5}$ cells/well. The next day, the HEK 293 cells were transfected with pcDNA3.1-hIDO or pcDNA3.1-hTDO using Lipofectamine 2000 according to the manufacturer's instructions. The cells were seeded in 96-well plates at a density of $2.5 \times 10^{4}$ cells/well $24 \mathrm{~h}$ after transfection and treated with tested compounds. After an additional $12 \mathrm{~h}$ of incubation, $200 \mu \mathrm{L}$ of the culture medium from each well was transferred to a 96 -well plate and mixed with $100 \mu \mathrm{L}$ of $30 \%$ trichloroacetic acid. The plate was incubated at $65^{\circ} \mathrm{C}$ for $15 \mathrm{~min}$ to hydrolyze the $\mathrm{N}$-formylkynurenine produced by the catalytic reaction of IDO1 or TDO2. The reaction mixture was then centrifuged at $12000 \mathrm{r} / \mathrm{min}$ for $10 \mathrm{~min}$. Then, $100 \mu \mathrm{L}$ of the supernatant was transferred to another 96-well plate and mixed with $100 \mu \mathrm{L}$ of $2 \%(\mathrm{w} / \mathrm{v}) p$-dimethylaminobenzaldehyde in acetic acid. The yellow color derived from kynurenine was measured at $492 \mathrm{~nm}$ using a SpectraMax Plus 384 microplate reader (Molecular Devices, Sunnyvale, CA, USA).

Determination of the inhibition kinetics of IDO1 inhibitors The inhibition kinetics were determined as described previously [20]. In brief, the enzymatic activity of IDO1 was determined with a serial dilution of the test compounds with L-tryptophan at concentrations ranging from $7.5 \mu \mathrm{M}$ to $30 \mu \mathrm{M}$. A function of $S / V$ against I was plotted, where $S$ represents the substrate concentration, $V$ represents the reaction velocity and $I$ represents the inhibitor concentration. The intersect points in the plots were used to measure an apparent inhibition constant, $K_{\mathrm{i}}$. Graphing and statistical analysis of the data were performed using Prism 4 software (GraphPad Software Inc., San Diego, CA, USA).

\section{Measurement of IDO1 absorbance spectra}

The absorbance spectra $(300-700 \mathrm{~nm})$ were measured immediately after the addition of the test compounds $(20 \mu \mathrm{M}$ in $0.1 \%$ dimethyl sulfoxide; DMSO) to rhIDO1 (3 $\mu \mathrm{M})$ in phosphate buffer using a SYNERGY H1 microplate reader (Bioteck Winooski, VT, USA). The ferrous form of heme was obtained by adding sodium dithionite $(10 \mathrm{mM})$. Changes in the absorbance at 404 or $428 \mathrm{~nm}$ indicated binding to the ferric or ferrous iron of the heme, respectively.

\section{Cell viability assay}

Cell viability was determined using a Cell Counting Kit-8 (CCK8) assay. HEK 293 cells were seeded in 96-well plates at a density of $2.5 \times 10^{4}$ cells/well, incubated for $24 \mathrm{~h}$, and then treated with the tested compounds. After an additional incubation of $12 \mathrm{~h}$, CCK8 was added to each well. After another $2 \mathrm{~h}$ incubation period, the absorbance was measured at $450 \mathrm{~nm}$ (SpectraMax Plus 384 

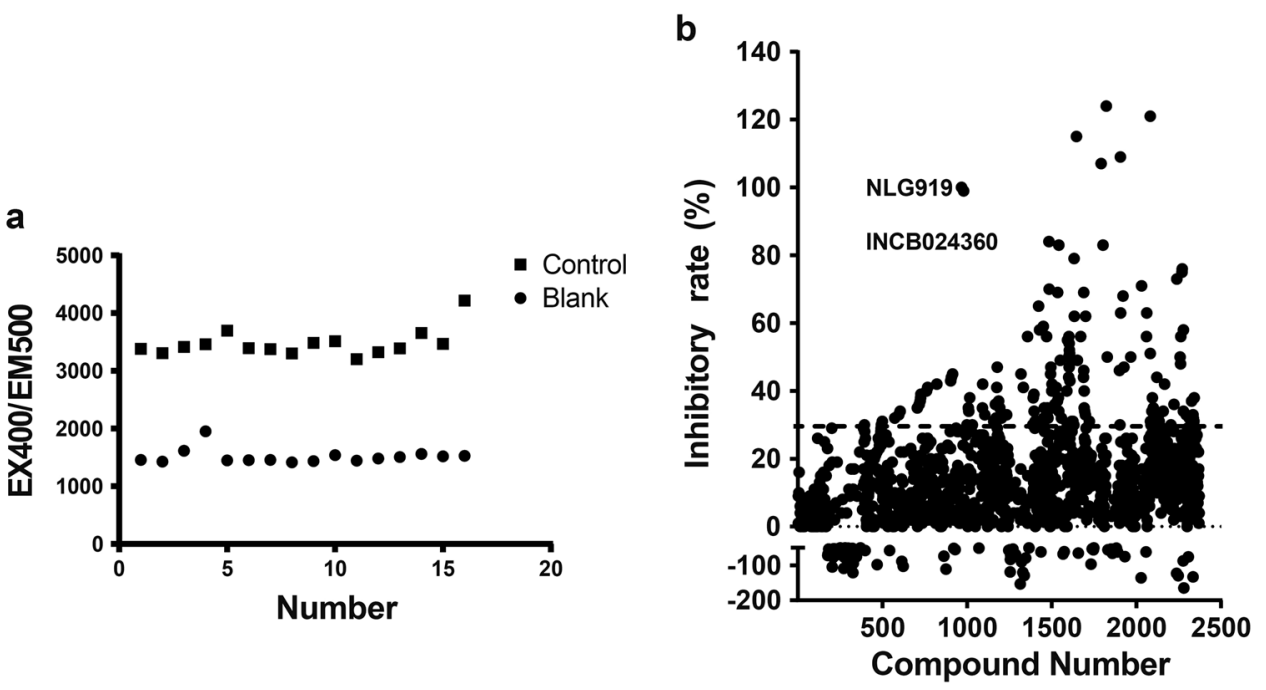

Fig. 1 Confirmation of the high-throughput screening assay. a $O D$ values of the blank and control. The $Z^{\prime}$ factor was determined to be 0.572 . b Fluorescence-based screening of Selleck Customized Library-Z100688 compounds $(n=2430)$ at $20 \mu$ M. NLG919 and INCB024360 are known IDO1 inhibitors

microplate reader, Molecular Devices). Graphing and statistical analysis of the data were performed using Prism 4 software (GraphPad Software Inc., San Diego, CA, USA).

\section{Molecular docking}

LigPrep (version 2.4, Schrödinger, LLC, New York, NY, 2010) was used to generate stereoisomers and tautomers, and the protonation states of the ligands at $\mathrm{pH} 7.0 \pm 2.0$ were determined with Epik [21]. For other parameters, the default values were used.

The crystal structure of IDO1 complexed with NLG919 analog 1CE (PDB access code: 5EK3) [22, 23] was selected for molecular docking. The structure was prepared with the Protein Preparation Wizard Workflow provided in the Maestro module of Schrödinger software (Schrödinger, LLC: New York, NY, 2015). The protein structure was first fixed by assigning bond orders, adding hydrogens, creating zero-order bonds to metals, creating disulfide bonds, filling in missing side chains using Prime, deleting water molecules $>5 \AA$ from the het group, removing waters with less than three $\mathrm{H}$-bonds to nonwaters, and restraining the minimization to allow only hydrogen atoms to be freely minimized. Subsequently, the receptor grid of the complex was generated with the Glide module of Schrödinger software, and the grid boxes were defined as a $10 \times 10 \times 10 \AA^{3}$ region centered at the original ligand of the complex structure. In addition, one metalligand interaction was introduced into the heme iron during grid box generation. For the other parameters, the default values were used.

\section{RESULTS}

Screening of the natural product library for inhibitors of rhIDO1 To identify novel IDO1 inhibitors with diverse structures, we used a fluorescent assay for measuring IDO1 activity that was suitable for high-throughput screening [19]. The $Z^{\prime}$ factor was determined to be 0.572 , confirming the stability of the high-throughput screening assay (Fig. 1a). A customized Library-Z100688 containing 2430 drug candidates targeting different oncogenic processes was screened as a pilot study. As shown in Fig. 1b, NLG919 and INCB024360, two known IDO1 inhibitors, displayed inhibitory activity against IDO1, indicating the sensitivity of this fluorescence-based assay. We next sought to screen an in-house natural product library containing 2000 natural products with this assay. A total of 79 compounds displayed inhibitory activity $\geq$
$30 \%$ against IDO1 at $20 \mu \mathrm{M}$ (Fig. 2a). Because compounds with fluorescence interfere with the analysis of kynurenine using fluorescence spectroscopy, the 79 compounds were re-evaluated with a pDMAB-based assay, in which kynurenine reacts with $p$-dimethylaminobenzaldehyde to produce a substance with an absorbance at $480 \mathrm{~nm}$. As shown in Fig. $2 \mathrm{~b}, 9$ of the 79 natural products were found to inhibit rhIDO1 by $>30 \%$. To further confirm the activities of these nine compounds against IDO1, HEK 293 cells overexpressing human IDO1 were treated with the nine natural products, and the cellular activity of IDO1 was detected by measuring the level of kynurenine released by the cells. Compounds 2, 7, and $\mathbf{8}$ inhibited the cellular activity of IDO1 by $79.7 \%, 101.8 \%$, and $83.6 \%$, respectively (Fig. 2c). Thus, compounds $\mathbf{2}, \mathbf{7}$, and $\mathbf{8}$ were identified as new inhibitors of IDO1.

Activity of compounds 2,7 , and 8 against rhIDO1 and cellular IDO1 We found that natural products $\mathbf{2}, \mathbf{7}$, and $\mathbf{8}$ were able to inhibit the activity of IDO1. As shown in Fig. 3a, compound 2, 9-Odemethyltrigonostemone, was isolated from the roots of Trigonostemon chinensis and has been reported to show cytotoxicity in multiple types of cancer cells [24]. Compound 7 is shikonin from Radix Arnebiae and was reported to possess anti-inflammatory activity [25]. Compound $\mathbf{8}$ is dihydrotanshinone I from Radix Salviae Miltiorrhizae and shows cytotoxicity against many types of cancer cells [26].

We next sought to examine the activity of compounds $\mathbf{2}, \mathbf{7}$, and 8 against IDO1 in more detail. These compounds inhibited the catalytic activity of IDO1 in a dose-dependent manner, with $I C_{50}$ values of $2.2 \mu \mathrm{M}, 0.98 \mu \mathrm{M}$, and $2.8 \mu \mathrm{M}$ in the case of compounds $\mathbf{2}$, 7, and 8, respectively (Fig. 3b). INCB024360 was included in the assay as a positive control, and it showed an $\mathrm{IC}_{50}$ of $0.11 \mu \mathrm{M}$. We next examined the ability of compounds $\mathbf{2}, \mathbf{7}$, and $\mathbf{8}$ to inhibit the catalytic activity of IDO1 in a cellular context by determining the level of kynurenine, the catabolite of tryptophan, in the cell culture media. As shown in Fig. 3c, compounds 2, 7, and $\mathbf{8}$ downregulated the level of kynurenine with $\mathrm{IC}_{50}$ values of $18.4 \mu \mathrm{M}, 2.2 \mu \mathrm{M}$, and $25.7 \mu \mathrm{M}$, respectively. The discrepancy between the $\mathrm{IC}_{50}$ values obtained in the biochemical assay and cellular assay might be owing to the ability of each compound to enter the cells. To determine whether the decrease in kynurenine production observed in cells treated with the compounds was owing to cytotoxicity, a CCK8 assay was performed. This assay showed that 

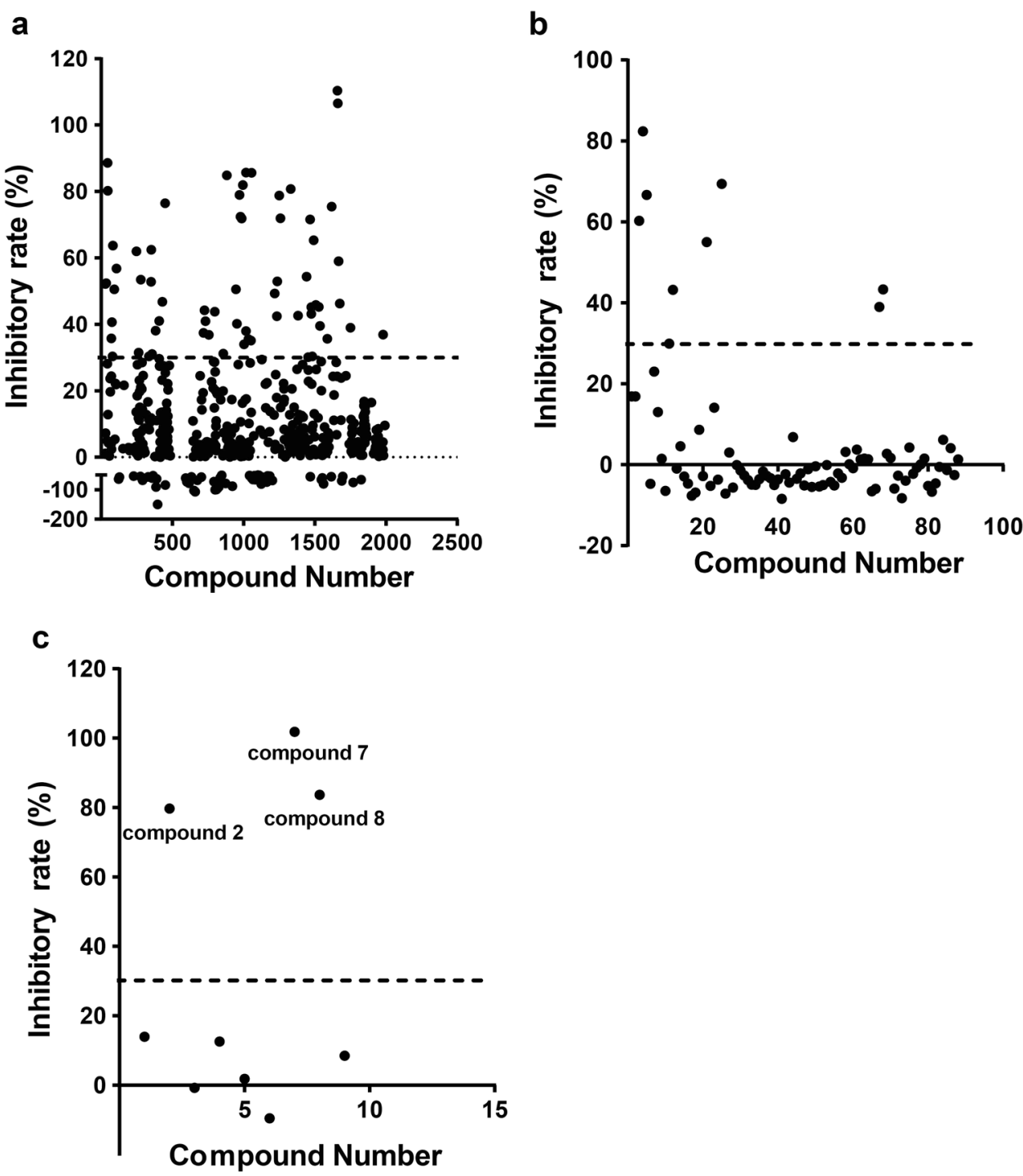

Fig. 2 Screening of the natural product library for inhibitors of recombinant human IDO1. a Screening of the natural product library $(n=$ $2000)$ at $20 \mu \mathrm{M}$. b The effects of 79 natural products on the activity of IDO1 using a method based on Ehrlich's reagent. c The effects of the nine selected compounds on the activity of IDO1 in HEK 293 cells expressing human IDO1. The dotted line indicates inhibition by $30 \%$

compounds $\mathbf{2}$ and $\mathbf{8}$ exhibited no effect on cell viability under the conditions used for the kynurenine assay. Compound 7 slightly reduced cell viability, but the effect was marginal compared with its activity against kynurenine production.

Compound $\mathbf{8}$ is a tanshinone analog and showed promising inhibition of IDO1 with low cytotoxicity. The three other tanshinone derivatives in the screened natural compound library displayed variable activities against IDO1 (Table 1), indicating that tanshinone derivatives may be promising candidates as IDO1 inhibitors.

Analysis of the inhibition modes

To elucidate their modes of inhibition, detailed kinetic experiments were performed with natural products $\mathbf{2}, \mathbf{7}$, and $\mathbf{8}$. The IDO1-mediated enzymatic assay was performed in the presence of the compounds with increasing concentrations of the substrate (tryptophan), and the inhibitory kinetics were analyzed by plotting $[S] /[V]$ against the concentration of the test compound, where [S] represents the concentration of tryptophan and [V] represents the reaction velocity [27]. For competitive inhibition, the plots of [S]/ [V] vs. [I] have no intersect [27], and consistent with previous publications [28], INCB024360 exhibited competitive inhibition (Fig. 4a). The plots corresponding to compound $\mathbf{2}$ matched plots typical of noncompetitive inhibition as they showed an $\mathrm{X}$ intersect (Fig. 4b), whereas the plots corresponding to compounds $\mathbf{7}$ and $\mathbf{8}$ were a good graphical match to uncompetitive inhibition (Fig. 4c, d). The $K_{\mathrm{i}}$ values of $\mathbf{2}, \mathbf{7}$, and $\mathbf{8}$ were calculated from the plots and were $6.9 \mu \mathrm{M}, 1.4 \mu \mathrm{M}$, and $2.8 \mu \mathrm{M}$, respectively.

Spectroscopic studies on the binding of compounds 2, 7, and 8 with IDO1

To provide evidence for the involvement of interactions of heme in the inhibition of IDO1 activity, we measured the absorbance of IDO1 in the presence of the ferric or ferrous forms after incubation with compounds 2, 7, 8, or INCB024360. The UV-Vis absorption properties of the porphyrin ring are highly sensitive and useful in understanding the ligand/substrate's ability to bind the IDO1 enzyme [18, 29-31]. The absorption spectrum of ferric-IDO1 showed a Soret peak at $404 \mathrm{~nm}$, which is in accordance with previously reported results [18, 30-33]. The absorbance of ferricIDO1 at $404 \mathrm{~nm}$ decreased in the presence of compounds $\mathbf{7}$ and 8 compared with the control, whereas the absorbance of ferrous- 
a<smiles>COc1cc2cc(C(C)(C)C)c(OC)cc2cc1C</smiles>

\section{Compound 2}

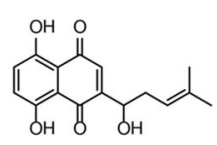

Compound 7

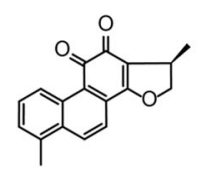

Compound 8 b
ID01

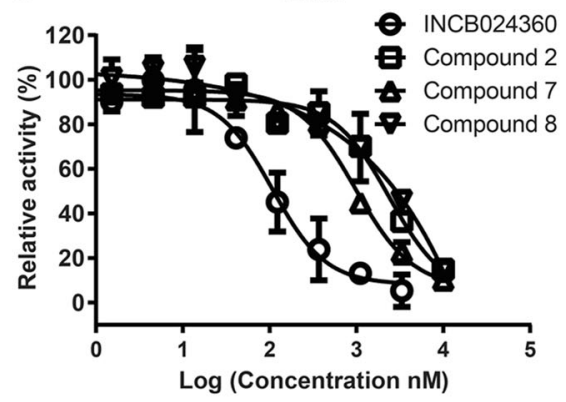

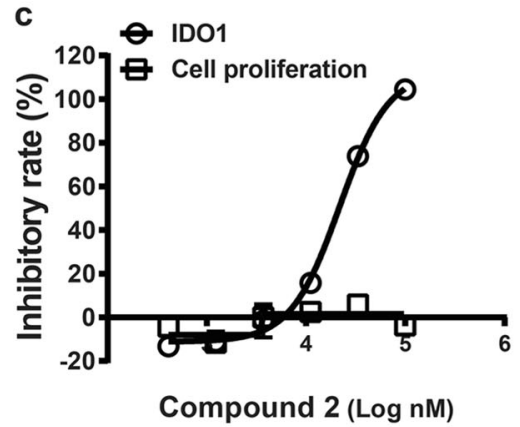
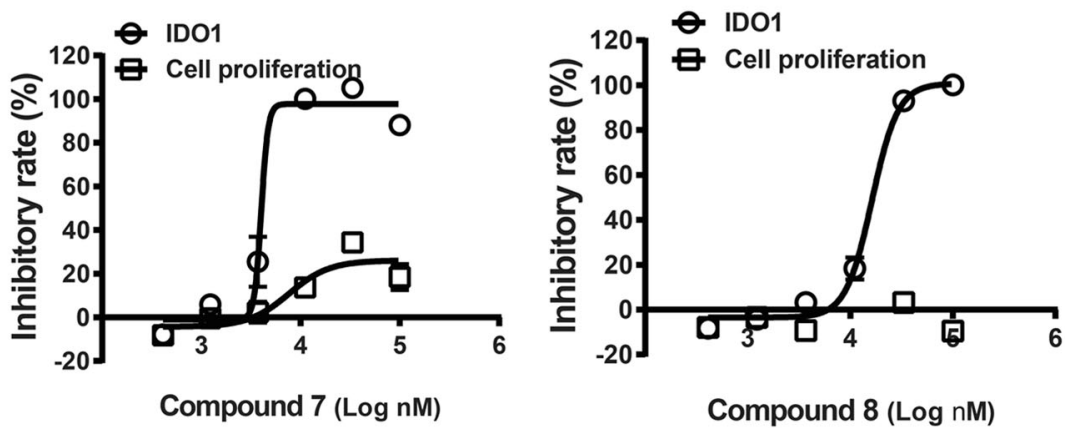

Fig. 3 The activities of compounds $\mathbf{2}, \mathbf{7}$, and $\mathbf{8}$ against rhIDO1 and cellular IDO1. a The structures of compounds $\mathbf{2}, \mathbf{7}$, and $\mathbf{8}$. $\mathbf{b}$ The effects of compounds 2, 7, 8, and INCB024360 on the activity of IDO1 based on the reaction of Ehrlich's reagent. c The effects of the test compounds on the cellular activity of IDO1 in HEK 293 cells. A CCK8 assay was performed to measure the cell viability at the end of treatment. Data shown are the mean \pm SD of biological triplicates from a representative experiment

Table 1 IDO1 inhibitory activity of tanshinone derivatives

Compound
B06E2 (Tanshinone II A)
B06F8 (Tanshinone I)
Compound 8 (Dihydrotanshinone I)

N.I. no inhibition

IDO1 remained unchanged under the same conditions, indicating that compounds $\mathbf{7}$ and $\mathbf{8}$ appeared to interact with the ferric form of IDO1 (Fig. 5a, b). The absorbances of ferric IDO1 at $404 \mathrm{nM}$ and ferrous IDO1 at $428 \mathrm{~nm}$ increased in the presence of compound $\mathbf{2}$, which might be owing to the fluorescent interference of compound 2.
Selectivity of compounds 2, 7, and 8 for IDO1 and TDO2

TDO2 is the other enzyme that also catalyzes the rate-limiting step of the kynurenine pathway in the liver. To determine their selectivity between IDO1 and TDO2, we measured the inhibitory activities of the selected compounds against purified IDO1 and TDO2. As shown in Fig. 6a, these fused heterocyclic compounds 

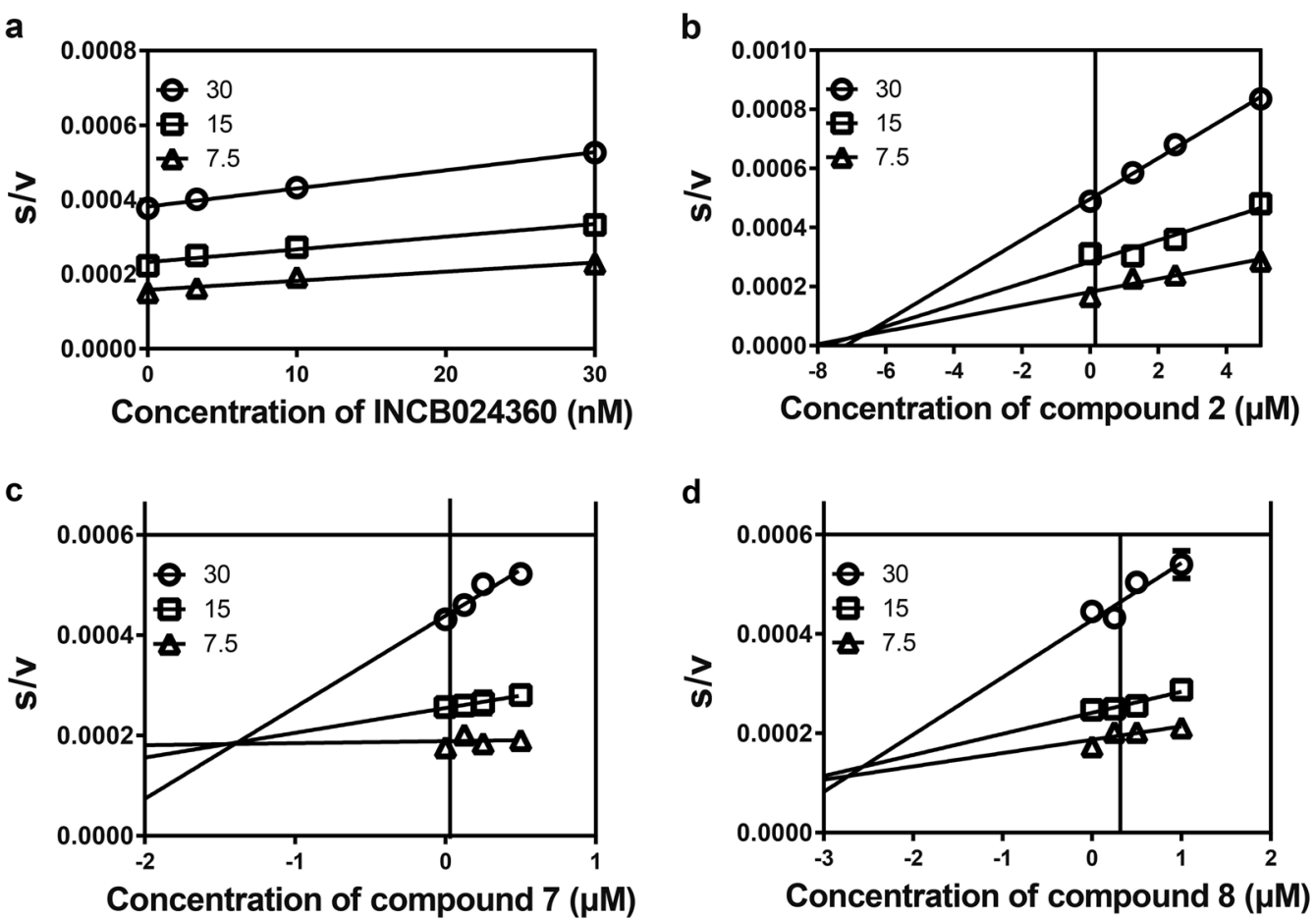

Fig. 4 Determination of the inhibition modes and kinetic parameters of compounds $\mathbf{2}, \mathbf{7}$, and $\mathbf{8}$. The IDO1 activities were determined in the presence of serially diluted INCB024360 a, compound $\mathbf{2}$ b, compound $\mathbf{7}$ c, or compound $\mathbf{8} \mathbf{d}$ with L-tryptophan concentrations varying from $7.5 \mu \mathrm{M}$ to $30 \mu \mathrm{M}$. [S]/[V] was plotted against [I]. The data presented are the mean \pm SD of biological triplicates from a representative experiment

possessed variable activities against TDO2. The $\mathrm{IC}_{50}$ values of compounds $\mathbf{2}, \mathbf{7}$, and $\mathbf{8}$ against purified TDO2 enzyme varied from 5 to $91 \mu \mathrm{M}(91.1 \mu \mathrm{M}, 5.05 \mu \mathrm{M}$, and $5.1 \mu \mathrm{M}$, respectively) (Fig. 6a). Compounds 2 and 7 were more potent against IDO1 (>10-fold) than TDO2, whereas compound $\mathbf{8}$ exhibited similar activity against IDO1 and TDO2. In a cellular context, compounds 2, 7, and 8 inhibited the activity of IDO1 and TDO2 with similar potencies, with $I_{50}$ values of $24.7 \mu \mathrm{M}, 1.4 \mu \mathrm{M}$, and $13.3 \mu \mathrm{M}$, respectively, against TDO2 (Fig. $6 \mathrm{~b}$ ). These results indicated that compounds $\mathbf{2}$, $\mathbf{7}$, and $\mathbf{8}$ were able to inhibit IDO1 and TDO2 in a cellular context.

Binding mode prediction

To explore the molecular basis of the inhibitory activity, molecular docking studies were performed. Compound $\mathbf{8}$ was docked into the binding pocket of a cocrystal structure of IDO1/1-CE $((1 \sim\{R\})-1-$ cyclohexyl-2-[(5 \{S\})-5 \{H\}-imidazo[1,5-b]isoindol-5-yl]ethanol, 1CE) (PDB access code: 5EK3) [23]. 1-CE is a NLG919 analog and known inhibitor of IDO1, and it displays a binding mode similar to that of compound $\mathbf{8}$ in the binding pockets based on the preliminary study. As shown in Fig. 7, compound 8 displays a binding mode similar to that of 1-CE, and the dihydrofuran oxygen of compound $\mathbf{8}$ coordinated with the heme iron. In binding pocket A, the naphthalene ring of compound 8 forms "T-shaped" $\pi-\pi$ interactions with Phe-163, and the major difference between the binding modes of these two compounds is in binding pocket $B$, where the 6-methyl-naphthalene group of compound $\mathbf{8}$ forms additional hydrophobic interactions.

Hit expansion and SAR analysis

Given that several tanshinone analogs were found to possess variable activities against IDO1, Hit expansion was conducted to explore the structure-activity relationships (Table 1). As shown in Table 1, B06F5 (cryptotanshinone) inhibited IDO1 by $>40 \%$ at $20 \mu \mathrm{M}$. According to the binding mode predicted by docking studies (Fig. 7), the dihydrofuran rings of compound 8 and B06F5 could form interactions with the heme iron, whereas B06E2 and B06F8 could not, which was in accordance with the result that compound 8 and B06F5 are active, whereas B06E2 and B06F8 are unable to inhibit IDO1. In addition, compound $\mathbf{8}$ was more active than B06F5, which might be owing to the additional $\pi-\pi$ interactions between the naphthalene ring of compound 8 and Phe-163. It is worth mentioning that although B06F8 may form "Tshaped" $\pi-\pi$ interactions with Phe-163, it cannot form interactions with the heme iron, which might explain its weak activity.

\section{DISCUSSION}

In this study, we identified compounds $\mathbf{2 ,} \mathbf{7}$, and $\mathbf{8}$ as novel IDO1 inhibitors by screening an in-house natural product library. These compounds inhibited IDO1 in the low micromolar range, with the most active (compound 7) displaying submicromolar activity. A detailed kinetic study revealed a noncompetitive inhibition profile for compound $\mathbf{2}$ and uncompetitive inhibition profiles for compounds $\mathbf{7}$ and $\mathbf{8}$. Compounds $\mathbf{7}$ and $\mathbf{8}$ have also been found to potentially bind to the ferric heme of IDO1. Compound $\mathbf{8}$ was identified as a derivative of tanshinone, and preliminary SAR analysis indicated that tanshinone derivatives may be promising hits for IDO1 inhibitors.

IDO1 inhibitors clinically developed to date are mainly tryptophan-competitive inhibitors (epacadostat), tryptophannoncompetitive inhibitors (navoximod) or irreversible inhibitors (BMS-986205). Compared with the existing IDO1 inhibitors, the inhibitors identified in this study exhibited novel chemical backbones. These compounds also displayed distinct modes of action against IDO1. For example, compound $\mathbf{2}$ exhibited noncompetitive inhibition, whereas compounds $\mathbf{7}$ and $\mathbf{8}$ showed uncompetitive inhibition and potentially bound to heme. Whereas uncompetitive inhibition requires the formation of an enzyme-substrate complex, noncompetitive inhibition can occur with or without the presence of the substrate. In particular, 

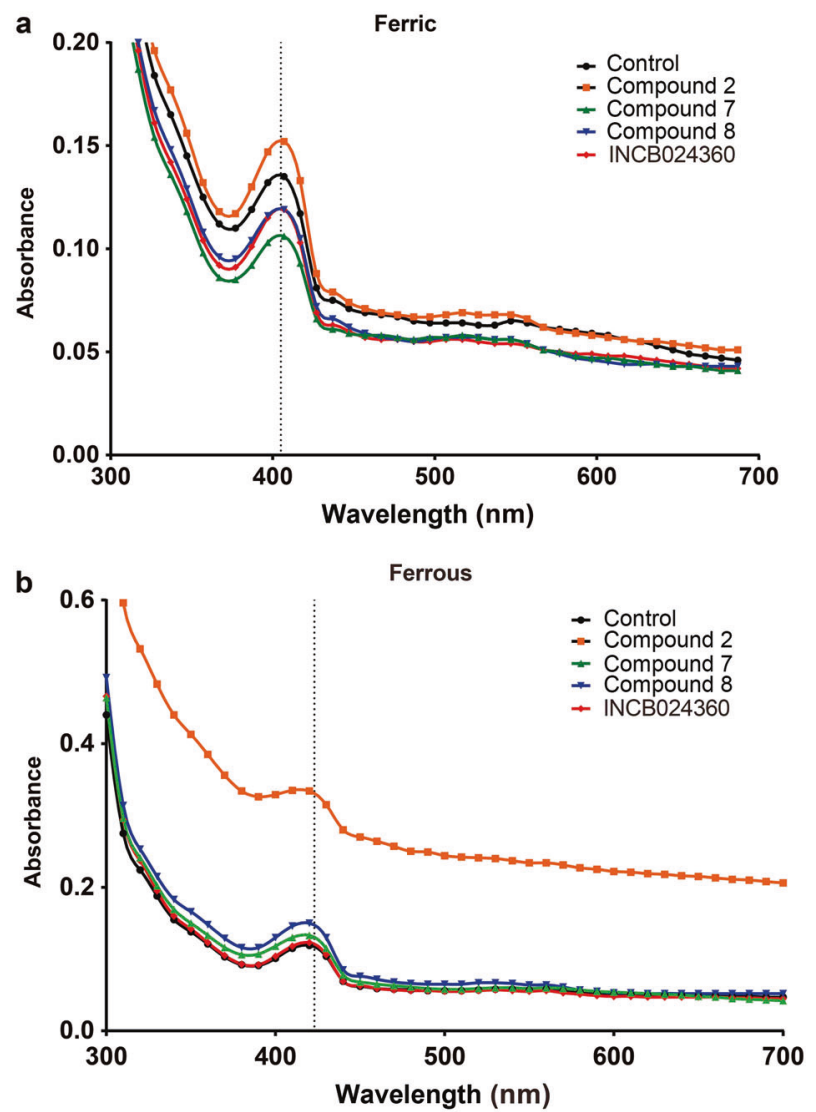

Fig. 5 Absorbance spectra of ferric or ferrous rhIDO1 in the presence of compounds $\mathbf{2}, \mathbf{7}$, and $\mathbf{8}$. Inset shows the absorbance difference spectra. a The absorbance of IDO1 ferric heme at $404 \mathrm{~nm}$ in the presence of compound $\mathbf{2}, \mathbf{7}$, or $8(20 \mu \mathrm{M})$. b The absorbance of IDO1 ferrous heme at $428 \mathrm{~nm}$ in the presence of compound $\mathbf{2 , 7}$, or $8(20 \mu \mathrm{M})$ compound 8 (dihydrotanshinone I) and several derivatives of tanshinone were found to display variable activities against IDO1. Interestingly, tanshinone derivatives were also identified as IDO1 inhibitors through a virtual screening [34], when this manuscript was in preparation. Tanshinone is a typical scaffold family derived from Danshen (a Chinese herb), the dried root of Salvia miltiorrhiza, which has been widely used for the treatment of cardiovascular and cerebrovascular diseases. These results supported that tanshinone derivatives were promising hits for the development of new IDO1 inhibitors.

Given that existing commonly used IDO1 inhibitors show limited effects in clinical trials [35], accumulating evidence indicates that the distinct tryptophan catabolic enzyme TDO2 may deplete tryptophan to mediate immune escape [20,36, 37]. TDO2 has been associated with increased grade, estrogen receptor-negative status, and reduced overall survival rates in triple negative breast cancer patients [38]. TDO2 has been reported to be overexpressed in multiple tumor types, including melanomas and brain and breast tumors [38, 39]. TDO2 inhibition has emerged as a parallel immunomodulatory strategy to attack tumors [40-44]. An early bioactive inhibitor, 680 C91 [40], has been tested in mouse studies, and it was able to reduce pulmonary metastasis in tumor-bearing mice. Recently, more potent and pharmacologically favorable compounds have been reported $[41-43,45]$. TDO2 is also a logical candidate to mediate resistance to IDO1-selective blockade. Therefore, IDO1/TDO2 dual inhibitors may achieve better efficacy. Natural products 2, 7, and $\mathbf{8}$ were found to inhibit IDO1 and TDO2 in cell-free and cellular contexts. Further study on the structure-activity relationship may lead to the development of potent IDO1/TDO2 dual inhibitors.

In summary, this study has identified compounds 2, 7, and 8 as novel IDO1/TDO2 inhibitors with new scaffolds via high-throughput screening of natural products. A kinetic study revealed the modes of inhibition and preliminary SARs of a group of tanshinone derivatives. This study provides new clues for the development of IDO1/TDO2 inhibitors with novel scaffolds.

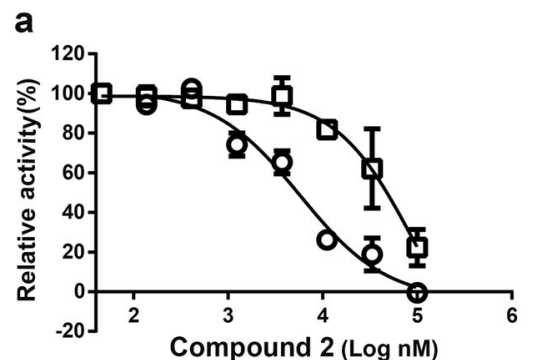

b

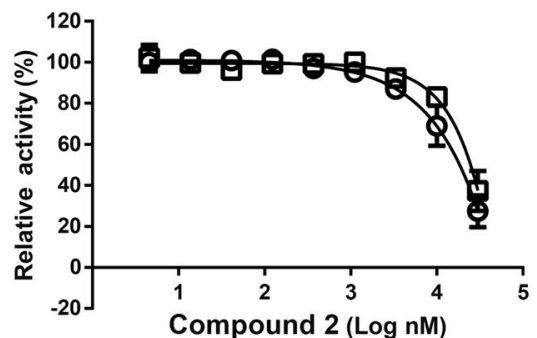

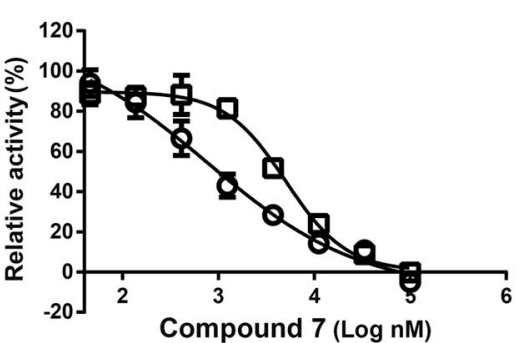

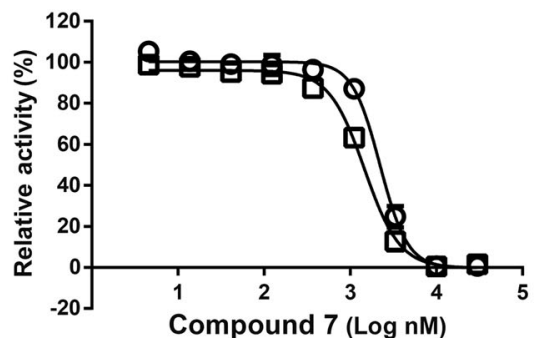

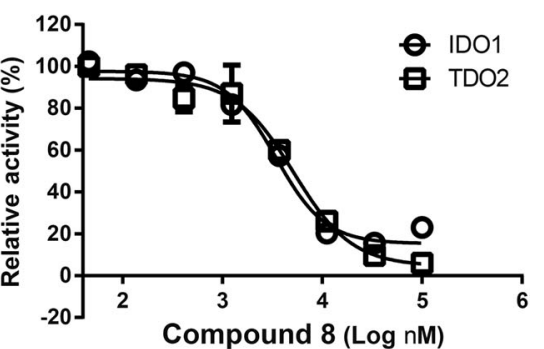

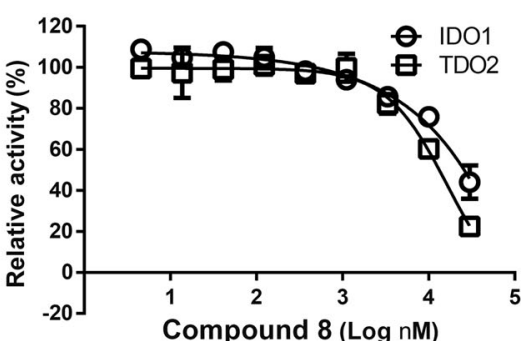

Fig. 6 Selectivity of compounds 2, 7, and $\mathbf{8}$ for IDO1 and TDO2. a The effects of compound 2, 7, or $\mathbf{8}$ on the activity of IDO1 and TDO2 with an Ehrlich's reagent-based assay. $\mathbf{b}$ The effects of compound $\mathbf{2}, \mathbf{7}$, or $\mathbf{8}$ on the activity of IDO1 and TDO2 in HEK 293 cells expressing human IDO1 or TDO2, respectively. Data are presented as the mean \pm SD of biological triplicates from a representative experiment 


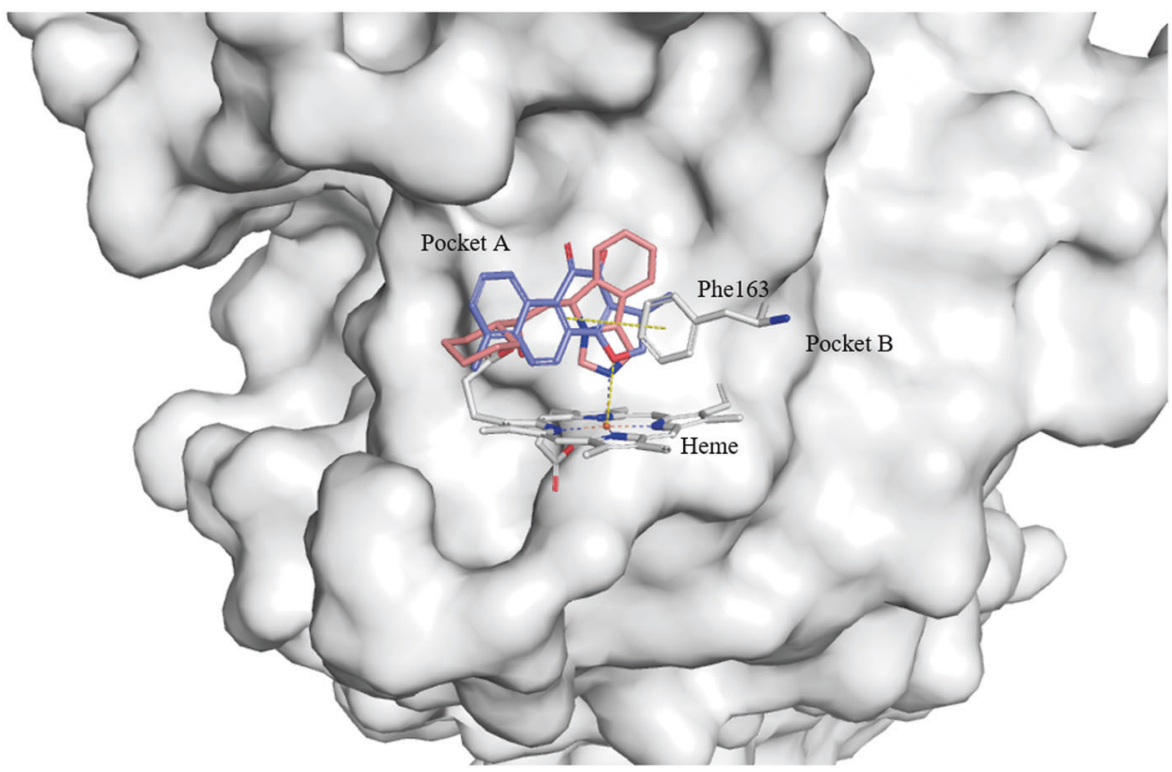

Fig. 7 Comparison of the binding modes of 1-CE (orange) and compound 8 (purple) in the active site of IDO1 (PDB ID: 5EK3), where the catalytic pocket of IDO1 is depicted as a gray surface. The image was generated using PyMOL, version 1.3

\section{ACKNOWLEDGEMENTS}

This work was supported by the National Natural Science Foundation of China (81773760, 81573305, and 81673327) and the International Partnership Program of the Chinese Academy of Sciences (153631KYSB20160004).

\section{AUTHOR CONTRIBUTIONS}

Conducted the experiments: WG, SY, PS and TBY. Contributed to the in-house natural products library or analytic tools: SY, CPT, TBY and MYZ. Performed the data analysis: WG, SY, PS, TBY and MYZ. Wrote or contributed to the writing of the manuscript: WG, SY, LHM, TBY and MYZ. Participated in the research design: LHM and YY.

\section{ADDITIONAL INFORMATION}

Competing interests: The authors declare no competing interests.

\section{REFERENCES}

1. Moffett JR, Namboodiri MA. Tryptophan and the immune response. Immunol Cell Biol. 2003;81:247-65.

2. Munn DH, Zhou M, Attwood JT, Bondarev I, Conway SJ, Marshall B, et al. Prevention of allogeneic fetal rejection by tryptophan catabolism. Science. 1998;281:1191-3.

3. Ball HJ, Jusof FF, Bakmiwewa SM, Hunt NH, Yuasa HJ. Tryptophan-catabolizing enzymes - party of three. Front Immunol. 2014; 5:485.

4. Okamoto A, Nikaido T, Ochiai K, Takakura S, Saito M, Aoki Y, et al. Indoleamine 2,3-dioxygenase serves as a marker of poor prognosis in gene expression profiles of serous ovarian cancer cells. Clin Cancer Res. 2005;11:6030-9.

5. Uyttenhove C, Pilotte L, Theate I, Stroobant V, Colau D, Parmentier N, et al. Evidence for a tumoral immune resistance mechanism based on tryptophan degradation by indoleamine 2,3-dioxygenase. Nat Med. 2003;9:1269-74.

6. Theate I, van Baren N, Pilotte L, Moulin P, Larrieu P, Renauld JC, et al. Extensive profiling of the expression of the indoleamine 2,3-dioxygenase 1 protein in normal and tumoral human tissues. Cancer Immunol Res. 2015;3:161-72.

7. Munn DH, Mellor AL. Indoleamine 2,3-dioxygenase and tumor-induced tolerance. J Clin Invest. 2007:117:1147-54.

8. Gajewski TF, Meng Y, Blank C, Brown I, Kacha A, Kline J, et al. Immune resistance orchestrated by the tumor microenvironment. Immunol Rev. 2006;213:131-45.

9. Bauer TM, Jiga LP, Chuang JJ, Randazzo M, Opelz G, Terness P. Studying the immunosuppressive role of indoleamine 2,3-dioxygenase: tryptophan metabolites suppress rat allogeneic T-cell responses in vitro and in vivo. Transpl Int. 2005;18:95-100.

10. Vottero E, Balgi A, Woods K, Tugendreich S, Melese T, Andersen RJ, et al. Inhibitors of human indoleamine 2,3-dioxygenase identified with a target-based screen in yeast. Biotechnol J. 2006;1:282-8.
11. Sugimoto $H$, Oda $S$, Otsuki $T$, Hino $T$, Yoshida $T$, Shiro $Y$. Crystal structure of human indoleamine 2,3-dioxygenase: catalytic mechanism of $\mathrm{O} 2$ incorporation by a heme-containing dioxygenase. Proc Natl Acad Sci U S A. 2006;103:2611-6.

12. Carr G, Tay W, Bottriell H, Andersen SK, Mauk AG, Andersen RJ. Plectosphaeroic acids $A, B$, and $C$, indoleamine 2,3-dioxygenase inhibitors produced in culture by a marine isolate of the fungus plectosphaerella cucumerina. Org Lett. 2009;11:2996-9.

13. Yue EW, Douty B, Wayland B, Bower M, Liu X, Leffet L, et al. Discover y of potent competitive inhibitors of indoleamine 2,3-dioxygenase with in vivo pharmacodynamic activity and efficacy in a mouse melanoma model. J Med Chem. 2009;52:7364-7.

14. Gaspari P, Banerjee T, Malachowski WP, Muller AJ, Prendergast GC, DuHadaway J, et al. Structure-activity study of brassinin derivatives as indoleamine 2,3-dioxygenase inhibitors. J Med Chem. 2006;49:684-92.

15. Kumar S, Jaller D, Patel B, LaLonde JM, DuHadaway JB, Malachowski WP, et al. Structure based development of phenylimidazole-derived inhibitors of indoleamine 2,3-dioxygenase. J Med Chem. 2008;51:4968-77.

16. John S, Thangapandian S, Sakkiah S, Lee KW. Identification of potent virtual leads to design novel indoleamine 2,3-dioxygenase inhibitors: pharmacophore modeling and molecular docking studies. Eur J Med Chem. 2010:45:4004-12.

17. Austin CJ, Mizdrak J, Matin A, Sirijovski N, Kosim-Satyaputra P, Willows RD, et al. Optimised expression and purification of recombinant human indoleamine 2,3dioxygenase. Protein Expr Purif. 2004;37:392-8.

18. Littlejohn TK, Takikawa O, Skylas D, Jamie JF, Walker MJ, Truscott RJ. Expression and purification of recombinant human indoleamine 2, 3-dioxygenase. Protein Expr Purif. 2000;19:22-9.

19. Tomek P, Palmer BD, Flanagan JU, Fung SP, Bridewell DJ, Jamie JF, et al. Formation of an $\mathrm{N}$-formylkynurenine-derived fluorophore and its use for measuring indoleamine 2,3-dioxygenase 1 activity. Anal Bioanal Chem. 2013:405:2515-24.

20. Voet D, Voet JG. Biochemistry. 3rd edn. New York: John Wiley \& Sons; 2001.

21. Shelley JC, Cholleti A, Frye LL, Greenwood JR, Timlin MR, Uchimaya M. Epik: a software program for $\mathrm{pK}$ a prediction and protonation state generation for druglike molecules. J Comput-Aided Mol Des. 2007;21:681-91.

22. Zhang H, Liu W, Liu Z, Ju Y, Xu M, Zhang Y, et al. Discovery of indoleamine 2,3dioxygenase inhibitors using machine learning based virtual screening. Medchemcomm. 2018;9:937-45.

23. Peng $\mathrm{YH}$, Ueng SH, Tseng CT, Hung MS, Song JS, Wu JS, et al. Important hydrogen bond networks in indoleamine 2,3-dioxygenase 1 (IDO1) inhibitor design revealed by crystal structures of imidazoleisoindole derivatives with IDO1. J Med Chem. 2016:59:282.

24. Seephonkai P, Sangdee A, Bunchalee P, Pyne SG. Cytotoxic and antiplasmodial compounds from the roots of Strophioblachia fimbricalyx. J Nat Prod. 2009;72:1892-4.

25. Zorman J, Suå JP, Hafner-Bratkoviä I. Shikonin suppresses NLRP3 and AIM2 inflammasomes by direct inhibition of caspase-1. PLoS One. 2016;11:e0159826. 
26. Li H, Zhang Q, Chu T, Shi HY, Fu HM, Song XR, et al. Growth-inhibitory and apoptosis-inducing effects of tanshinones on hematological malignancy cells and their structure-activity relationship. Anticancer Drugs. 2012;23:846-55.

27. Cornish-Bowden A. A simple graphical method for determining the inhibition constants of mixed, uncompetitive and non-competitive inhibitors. Biochem J. 1974;137:143-4.

28. Prendergast GC, Malachowski WP, DuHadaway JB, Muller AJ. Discovery of IDO1 inhibitors: from bench to bedside. Cancer Res. 2017;77:6795-811.

29. Dawson JH, Andersson LA, Sono M. Spectroscopic investigations of ferric cytochrome P-450-CAM ligand complexes. Identification of the ligand trans to cysteinate in the native enzyme. J Biol Chem. 1982;257:3606-17.

30. Rohrig UF, Majjigapu SR, Grosdidier A, Bron S, Stroobant V, Pilotte L, et al. Rationa design of 4-aryl-1,2,3-triazoles for indoleamine 2,3-dioxygenase 1 inhibition. J Med Chem. 2012;55:5270-90.

31. Schenkman JB, Sligar SG, Cinti DL. Substrate interaction with cytochrome P-450. Pharmacol Ther. 1981;12:43-71.

32. Malachowski WP, Winters M, DuHadaway JB, Lewis-Ballester A, Badir S, Wai J, et al. O-alkylhydroxylamines as rationally-designed mechanism-based inhibitors of indoleamine 2,3-dioxygenase-1. Eur J Med Chem. 2016;108:564-76.

33. Panda S, Roy A, Deka SJ, Trivedi V, Manna D. Fused heterocyclic compounds as potent indoleamine-2,3-dioxygenase 1 inhibitors. ACS Med Chem Lett. 2016;7:1167-72

34. Zhang H, Wei L, Liu Z, Ju Y, Xu MY, Yue Z, et al. Discovery of indoleamine 2,3-dioxygenase inhibitors using machine learning based virtual screening. Medchemcomm. 2018;9:937-45.

35. Companies Scaling Back IDO1 Inhibitor Trials. Cancer Discovery. 2018;8:OF5-OF.
36. van Baren N, Van den Eynde BJ. Tumoral immune resistance mediated by enzymes that degrade tryptophan. Cancer Immunol Res. 2015;3:978-85.

37. Platten M, Wick W, Van den Eynde BJ. Tryptophan catabolism in cancer: beyond IDO and tryptophan Depletion. Cancer Res. 2012;72:5435-40.

38. D'Amato NC, Rogers TJ, Gordon MA, Greene LI, Cochrane DR, Spoelstra NS, et al. A TDO2-AhR signaling axis facilitates anoikis resistance and metastasis in triplenegative breast cancer. Cancer Res. 2015;75:4651-64.

39. Opitz CA, Litzenburger UM, Sahm F, Ott M, Tritschler I, Trump S, et al. An endogenous tumour-promoting ligand of the human aryl hydrocarbon receptor Nature. 2011;478:197-203.

40. Salter M, Hazelwood R, Pogson $\mathrm{Cl}$, lyer R, Madge DJ. The effects of a novel and selective inhibitor of tryptophan 2,3-dioxygenase on tryptophan and serotonin metabolism in the rat. Biochem Pharmacol. 1995;49:1435-42.

41. Pilotte L, Larrieu P, Stroobant V, Colau D, Dolusic E, Frederick R, et al. Reversal of tumoral immune resistance by inhibition of tryptophan 2,3-dioxygenase. Proc Natl Acad Sci U S A. 2012;109:2497-502.

42. Georgios P, Mowat CG. Antitumour agents as inhibitors of tryptophan 2,3-dioxygenase. Biochem Biophys Res Commun. 2014;443:28-31.

43. Wu JS, Lin SY, Liao FY, Hsiao WC, Lee LC, Peng YH, et al. Identification of substituted naphthotriazolediones as novel tryptophan 2,3-dioxygenase (TDO) inhibitors through structure-based virtual screening. J Med Chem. 2015;58:7807-19.

44. Abdel-Magid AF. Targeting the inhibition of tryptophan 2,3-dioxygenase (TDO-2) for cancer treatment. ACS Med Chem Lett. 2016;8: 11-13.

45. Dolusic E, Larrieu P, Moineaux L, Stroobant V, Pilotte L, Colau D, et al. Tryptophan 2,3-dioxygenase (TDO) inhibitors. 3-(2-(pyridyl)ethenyl)indoles as potential anticancer immunomodulators. J Med Chem. 2011;54:5320-34. 